\title{
Neuropsychological and neuroimaging markers in prediction of cognitive impairment after ischemic stroke: a prospective follow-up study
}

This article was published in the following Dove Press journal:

Neuropsychiatric Disease and Treatment

16 October 2015

Number of times this article has been viewed

\author{
Shima Mehrabian' \\ Margarita Raycheva' \\ Neli Petrova ${ }^{2}$ \\ Armina Janyan ${ }^{3,4}$ \\ Mariya Petrova' \\ Latchezar Traykov' \\ 'Clinic of Neurology, University \\ Hospital Alexandrovska, Sofia, ${ }^{2} \mathrm{Clinic}$ \\ of Neurology, MHAT "Ruse", Ruse, \\ ${ }^{3}$ Research Center for Cognitive \\ Science, Department of Cognitive \\ Science and Psychology, New \\ Bulgarian University, Sofia, Bulgaria; \\ ${ }^{4}$ Laboratory for Cognitive Studies in \\ Language, National Research Tomsk \\ State University, Tomsk, Russia
}

Background: There are few longitudinal studies with controversial results examining delayed changes in cognition after ischemic stroke and predictive values of neuropsychological and neuroimaging markers.

Objective: The objectives of this study were to evaluate the delayed changes in cognition in poststroke patients and their relationship to the neuropsychological and neuroimaging markers measured during the acute poststroke phase.

Methods: Eighty-five first-ever stroke inpatients (mean age 65.6 \pm 5.6 years) without previous cognitive complaints were prospectively evaluated with a comprehensive neuropsychological battery at the 5 th day and the $1 \mathrm{st}, 6$ th, and 12 th months. A wide range of clinical, radiological, and neuropsychological variables were examined.

Results: Our results showed significantly poorer performance on mini-mental state examination, memory, attention/executive functions, and processing speed in patients with stroke in comparison with stroke-free cognitively intact controls. Multiple regression analysis revealed that hippocampal atrophy is the strongest predictor of delayed cognitive impairment. Secondary divided subgroups according to Isaacs Set Test (IST) score showed that patients with IST score $\leq 28$ had different patterns of cognitive and neurological impairment after 1 year. Baseline impairments in attention/executive functions and memory were associated with development of dementia in poststroke patients.

Conclusion: Executive functioning deficit appears to have a predictive power for cognitive impairment progression. The study suggests that IST as a screening test has a potential to be a reliable and quick tool for poststroke cognitive impairment evaluation and delayed cognitive and neurological outcome. Hippocampal atrophy was the strongest predictor for cognitive impairment outcome, even in poststroke cognitive impairment. The findings may set the stage for better poststroke management.

Keywords: hippocampal atrophy, IST, neuropsychological predictors, poststroke dementia

\section{Introduction}

Poststroke cognitive impairment is frequent. There are, however, few longitudinal studies examining delayed changes in cognition, and predictive values of neuropsychological and neuroradiological markers. ${ }^{1-5}$ In community-based studies, the prevalence of poststroke dementia (PSD) is approximately $30 \%$ and the incidence of new-onset dementia after stroke increases from $7 \%$ after 1 year to $48 \%$ after 25 years. ${ }^{6}$ Some studies indicate that PSD may be reversible in a substantial proportion of patients. ${ }^{5}$ In contrast, other studies found that not only is the risk of PSD high immediately after stroke but it also remains higher in nondemented patients 3 months after the stroke. ${ }^{1}$ There is a need to identify individuals at particular risk of poststroke cognitive decline,
Correspondence: Latchezar Traykov Clinic of Neurology, University Hospita Alexandrovska, I Georgi Sofiiski Street, 143| Sofia, Bulgaria

Tel +35929230752

Fax +35929520427

Email traykov_ı@yahoo.fr 
and hence to target interventions for secondary prevention and improve the outcome and quality of life of stroke patients.

Identifying poststroke cognitive impairment at an early stage might increase the possibility of reducing or even preventing further cognitive decline. Few studies have examined the prognostic value of these impairments at a poststroke early stage. ${ }^{7}$ Identifying a quick neuropsychological screening tool which could predict delayed cognitive and functional decline in stroke patients is a challenge. The role of neuroimaging markers including white matter changes (WMC) and medial temporal lobe atrophy (MTLA) as a contributor to poststroke cognitive decline is still controversial. ${ }^{8}$

We present results from a prospective, hospital-based study of consecutive, first-ever stroke patients, designed to evaluate the delayed changes in cognition and their relationship to neuropsychological and neuroimaging markers. The study aimed to determine a quick cognitive screening test and neuroimaging markers measured in the acute poststroke phase which can accurately predict delayed cognitive and functional decline in poststroke patients.

\section{Material and methods}

Included in this study were consecutive inpatients with a first-ever ischemic cerebral stroke, aged 50-80 years, within 3 days of stroke onset. Patients were recruited from the acute stroke unit of the Clinic of Neurology, Ruse, Bulgaria. Stroke was defined as acute onset of focal neurological deficits attributable to cerebrovascular disease and documented by computerized tomography (CT).

Inclusion criteria for the study were: 1) Bulgarian ethnicity; 2) well-documented clinical presentation and CT scan of the brain; 3) ability to give informed consent or the availability of relative/caregiver to give proxy consent to participate in the study; and 4) availability of caregiver to give informed knowledge of the patients' earlier and current cognitive functioning.

Exclusion criteria included: 1) patients with a primary hemorrhagic stroke (intracerebral or subarachnoid); 2) history of any preceding cerebral vascular event; 3) patients with National Institutes of Health Stroke Scale (NIHSS) score $>6$; 4) persistent disturbed level of consciousness; 5) persistent aphasia; 6) history of prestroke cognitive impairment; 7) brain ischemia due to cardiorespiratory arrest; 8) systemic diseases known to involve the central nervous system; 9) severe sensory impairment (blindness and deafness); 10) known malignant disease or other chronic disease with poor prognosis; 11) neurological conditions (ie, Parkinson's disease, epilepsy, and head injury); and
12) history of psychiatric conditions (ie, depression, alcohol, and drug dependence).

A standard stroke evaluation was conducted on 522 consecutive patients admitted to stroke unit: 85 met the selection criteria; eleven were lost for follow-up at 12 months.

We composed a control group of 25 normal control subjects (NCs), matched to the patients group according to their age and educational level. All subjects gave written informed consent for their participation in the study which was approved by the local ethics committee. The guidelines of the ethics committee were adhered to.

\section{Assessments and data collection}

A vascular neurologist conducted a thorough physical examination and reviewed the imaging data and medical records of potential participants to determine eligibility. Baseline demographic and clinical data were collected. Neurological and neuropsychological assessments were conducted by an experienced neurologist and neuropsychologist at baseline (5th day after stroke onset) and at 1st, 6th, and 12th months.

Demographic data including age, sex, and educational level were collected. All patients were subjected to cardiac examination that included an electrocardiogram and echocardiography of the heart. Assessment of neurological status was determined using NIHSS score. ${ }^{9}$

\section{Neuroimaging characteristics}

A non-contrast CT brain scan examination was done for all patients. For hippocampal rating, we used the scale described in detail by Scheltens et al. ${ }^{10,11}$ In brief, it is a five-point scale of hippocampal atrophy (normal $=0$, severe atrophy $=4$ ). For periventricular changes (PVCs) and deep WMC, we used the scale of Scheltens ${ }^{12}$ evaluating the presence and extent of PVCs and deep WMC in different anatomic regions with a 0 -to-6-point scale. Wattjes et al ${ }^{13}$ described excellent intraobserver agreement between $\mathrm{CT}$ and magnetic resonance imaging (MRI) for medial temporal atrophy (mean $\kappa, 0.88$ and 0.86 on the left and right sides of the brain, respectively) and substantial overall agreement concerning WMC (mean $\kappa, 0.79)$ with published visual rating scales.

\section{Cognitive and neuropsychiatry assessments}

A set of standardized neuropsychological tests sensitive for mild cognitive impairment (MCI) and covering major cognitive domains were selected. ${ }^{14}$

A detailed neuropsychological assessment was done four times for all patients, at the baseline ( 5 th day of stroke onset) 
and after 1, 6, and 12 months. Normative data for these tests were also established in the control group.

Several cognitive domains were assessed:

1) Global cognitive functioning using the standard minimental state examination (MMSE). In our study, we also used the scores of five MMSE subtests.

2) Learning and episodic verbal memory was assessed with list learning subtest from the Consortium to Establish a Registry for Alzheimer's Disease (CERAD) Neuropsychological Battery, using several measures: immediate recall (number of words recalled over three learning trials), delayed recall after 15 minutes delay, and recognition.

3) Attention was tested by the Trail Making Test, part A (TMT-A)

4) As a substantial part of executive functioning, we assessed cognitive flexibility/set shifting using TMT-B, and verbal fluency/set sifting using Isaacs Set Test (IST), ${ }^{15}$ 15 -second version. The IST assessed verbal fluency by measuring the ability to generate lists of words in four semantic categories (fruits, colors, cities, and animals) in a 15 -second interval. ${ }^{16}$ IST is considered as a brief screening timed cognitive test with highly loaded executive functioning (cognitive set shifting, generation, and processing speed) and semantic and working memory, found to be of predictive value for preclinical detection of dementia symptoms.

5) Language abilities were assessed by the 15 -item subset of the Boston Naming Test.

6) Constructive praxis was evaluated by means of 4 Figures Coping Subtest from CERAD Neuropsychological Battery. The psychiatric evaluation included a semistructured interview and the 15-item Geriatric Depression Scale (GDS). Presence of depression was considered in participants with a GDS score more than 4. Independence level of everyday functioning was evaluated by Instrumental Activities of Daily Living (IADL) ${ }^{17}$ with caregiver interview. Restriction in four IADL (telephone, transportation, medication, and finances) and depressive symptoms were examined at each follow-up visit.

In secondary analyses, we classified the stroke patients into two subgroups according to their IST score at baseline (groups at risk and normal limits [NL]) and compared their neuropsychological performance. We studied the pattern of neurological and cognitive changes in follow-up examinations in secondary subgroups.

The clinical and neuropsychological assessment of patients also included a diagnosis of no cognitive impairment (NCI), $\mathrm{MCI}$, or presence of dementia at each time-point examination.
MCI was diagnosed using Winblad et al criteria ${ }^{18}$ and objective cognitive impairment in one or more cognitive domains with a score of 1.0 standard deviation (SD) below the mean from the age-matched control population, while dementia was diagnosed using the Diagnostic and Statistical Manual of Mental Disorders, 4th edition criteria ${ }^{19}$ and National Institute of Neurological Disorders and Stroke and Association Internationale pour la Recherche et l'Enseignement en Neurosciences (NINDS-AIREN) criteria.

\section{Prospective follow-up assessments after I, 6, and 12 months}

All subjects were prospectively followed-up through the outpatient neurology clinic at 1st, 6th, and 12th months after stroke onset. Patients were similarly assessed, using the same battery of neuropsychological assessment, NIHSS, GDS, and IADL scale.

\section{Statistical analysis}

Data were expressed as mean $\pm \mathrm{SD}$. Calculations were done with the statistical package STATISTICA for Windows (version 8). A chi-square test was used for the group comparisons of qualitative variables and a one-way analysis of variance (ANOVA) or $t$-tests were performed for quantitative variables. Repeated measures ANOVA (rANOVA) was applied to trace the level of cognitive functions in time together with the ability of assessment types to differentiate between groups divided by IST score. Thus, only results with interactions were valued. Bonferroni post hoc test was applied when needed. Multiple regression analyses were used to assess the neuroimaging predictors for cognitive decline.

For all tests, values of $P<0.05$ were considered statistically significant.

\section{Results}

Patients and control demographic information is presented in Table 1. There was no difference in age or education level between strokes and controls. The final sample included 85 patients, 67 males and 18 females, with mean age $65.6 \pm 5.6$ years (range: $50-80$ years). The distribution of stroke lesions showed left middle cerebral artery lesions in 29 subjects, right middle cerebral artery lesions in 39 subjects, and vertebrobasilar lesions in 17 subjects. The mean score of NIHSS was $4.6 \pm 0.9$ at the acute phase of stroke at baseline. Mild depression was present in $36.4 \%$ of stroke patients at baseline.

Table 2 presents the neuropsychological performance in controls and patients at baseline (5th day after stroke onset) and follow-up assessments. Stroke patients had significantly 
Table I Baseline characteristics of patients and normal controls (NCs)

\begin{tabular}{lllll}
\hline Baseline & NCs N=25 & $\begin{array}{l}\text { Poststroke } \\
\text { patients N=85 }\end{array}$ & $\begin{array}{l}\text { Poststroke patients } \\
\text { (NL) (IST > 28) N=46 }\end{array}$ & $\begin{array}{l}\text { Poststroke patients } \\
\text { (at risk) (IST } \leq \mathbf{2 8}) \mathbf{N}=\mathbf{3 9}\end{array}$ \\
\hline Age, years & $64.1(5.3)$ & $65.6(5.6)$ & $64.5(6.0)$ & $66.6(5.4)$ \\
Sex (male/female) & $18 / 7$ & $67 / 18$ & $34 / 12$ & $33 / 6$ \\
Education, years & $11.7(2.2)$ & $11.3(2.2)$ & $11.5(2.4)$ & $11.0(1.8)$ \\
MMSE & $27.9(1.4)$ & $26.6(2.3)^{* * *}$ & $27.3(1.4)^{* *}$ & $25.7(2.7)$ \\
Diabetes (\%) & 4 & $28 *$ & 30 & 26 \\
Hypertension (\%) & 76 & 86 & 85 & 87 \\
NIHSS & 0 & $4.6(0.9)$ & $4.6(0.7)$ & $4.7(0.6)$ \\
\hline
\end{tabular}

Notes: Means are given with standard deviations in parentheses. A comparison (t-test) with control group was performed. $* P<0.05 ; * * P<0.0$ I; $* * * P<0.001$. Insignificant results are with $P>0.1$.

Abbreviations: IST, Isaacs Set Test; MMSE, mini-mental state examination; NCs, normal controls; NIHSS, National Institutes of Health Stroke Scale; NL, normal limits.

lower performance on most cognitive tasks at first evaluation. There were only two cognitive domains, language (Boston Naming Test) and praxis (figure copying), that were within the same range as controls.

The most significant differences between patients and NCs were found on tasks assessing orientation, executive functions, and memory. General cognitive functioning (MMSE) score at baseline assessment was significantly below the mean score of NCs. MMSE score was reduced because of mild anomia and orientation deficits in some of the scale items. Patients had significantly lower scores on all measures of verbal learning. Their performance was characterized by reduced short-term memory, slow learning, and lowered level of recollection from long-term memory. Pattern of memory impairment resembled the one observed in medial temporal lobe dysfunction. The most significantly impaired were all timed tests of executive functioning - IST and TMT (A and B). All patients at baseline were cognitively slow, had noneffective set shifting, and reduced semantic generation.

Follow-up assessments revealed some changes in pattern of impairments, but the general cognitive functioning (MMSE) and executive functioning remained at significantly lower level of performance compared with NCs. Although patients gained better results in the 6th month assessment, they got worse results on cognitive testing as well as on functional scale of daily living activities 1 year after the stroke.

Table 2 Neuropsychological performance in NCs and patients at 5th day after stroke onset (baseline) and follow-up assessments

\begin{tabular}{|c|c|c|c|c|c|}
\hline & NC & $\begin{array}{l}\text { Stroke patients } \\
\text { 5th day }\end{array}$ & $\begin{array}{l}\text { Stroke patients } \\
\text { Ist month }\end{array}$ & $\begin{array}{l}\text { Stroke patients } \\
\text { 6th month }\end{array}$ & $\begin{array}{l}\text { Stroke patients } \\
\text { I 2th month }\end{array}$ \\
\hline MMSE & $27.9(1.3)$ & $26.6(2.3)^{* *}$ & $26.3(2.8)^{* *}$ & $26.9(2.2)^{*}$ & $26.4(2.8)^{*}$ \\
\hline - Orientation & $10.0(0.0)$ & $9.7(0.6)^{*}$ & $9.6(1.0)^{*}$ & $9.7(0.7)^{*}$ & $9.6(0.8)^{* *}$ \\
\hline - Memory & $5.5(0.7)$ & $5.4(0.8)$ & $5.3(0.9)$ & $5.4(0.8)$ & $5.3(0.9)$ \\
\hline - Attention & $4.4(0.7)$ & $4.1(1.1)$ & $4.0(I .1)$ & $4.2(1.0)$ & $4.0(1.3)$ \\
\hline - Language & $7.1(0.7)$ & $6.6(0.7)^{* *}$ & $6.7(0.7)^{*}$ & $6.9(0.6)$ & $6.8(0.8)$ \\
\hline - Praxis & $0.9(0.3)$ & $0.8(0.4)$ & $0.7(0.5)$ & $0.8(0.4)$ & $0.7(0.4)$ \\
\hline IST & $34.8(3.1)$ & $28.4(5.9)^{* * *}$ & $28.5(6.3)^{* * *}$ & $30.0(5.7)^{* * * *}$ & $29.4(5.7)^{* * *}$ \\
\hline IADL (4 items) & $0(0)$ & $0.2(0.6)$ & $0.6(1.0)^{* *}$ & $0.5(0.9)^{* *}$ & $0.7(1.6)^{*}$ \\
\hline \multicolumn{6}{|c|}{ CERAD episodic memory } \\
\hline \multicolumn{6}{|l|}{ - Immediate recall } \\
\hline - I trial & $4.0(1.5)$ & $3.0(1.4)^{* *}$ & $4.3(1.5)$ & $4.7(1.7)^{\sim}$ & $4.6(1.7)^{\sim}$ \\
\hline - II trial & $6.6(1.1)$ & $5.1(1.6)^{* * *}$ & $5.8(1.6)^{*}$ & $6.0(1.7)$ & $6.1(1.7)$ \\
\hline - III trial & $7.3(1.1)$ & $6.0(1.6)^{* * *}$ & $6.3(1.9)^{* * *}$ & $6.3(1.6)^{* * *}$ & $6.6(1.6)^{* * *}$ \\
\hline - Delayed recall & $5.3(1.6)$ & $3.3(2.2)^{* * *}$ & $4.4(2.6)$ & $4.7(2.2)$ & $5.2(2.4)$ \\
\hline - Recognition & $18.7(1.3)$ & $16.6(2.8)^{* * *}$ & $17.9(2.3)^{\sim}$ & $18.0(2.3)$ & $18.3(2.0)$ \\
\hline TMT-A & $57.3(13.6)$ & $98(46)^{* * * *}$ & $86(4 I)^{* * *}$ & $80(38)^{* *}$ & $83(44)^{* *}$ \\
\hline TMT-B & II I.7 (27.3) & $209(73)^{* * *}$ & $\mid 88(8 \mid)^{* * *}$ & $174(74)^{* * *}$ & $173(77)^{* * *}$ \\
\hline BNT & I $4.6(0.6)$ & $14.1(1.1)^{\sim}$ & $14.5(0.9)$ & I $4.6(0.7)$ & I $4.6(0.7)$ \\
\hline Figure copying & $10.5(0.7)$ & $10.2(I . I)$ & $10.3(1.1)$ & $10.2(1.1)$ & $10.2(1.0)$ \\
\hline GDS & $3.4(2.0)$ & $4.2(3.2)$ & $4.2(3.1)$ & $3.8(3.4)$ & $3.8(3.5)$ \\
\hline
\end{tabular}

Notes: Means are given with standard deviations in parentheses. A comparison ( $t$-test or chi-square) with control group was performed for each test in follow-up examinations. $-P<0.09 ; * P<0.05 ; * * P<0.01 ; * * * P<0.00$ I. Insignificant results are with $P>0.1$.

Abbreviations: BNT, Boston Naming Test; CERAD, Consortium to Establish a Registry for Alzheimer's Disease; GDS, Geriatric Depression Scale; IADL, Instrumental Activities of Daily Living; IST, Isaacs Set Test; MMSE, mini-mental state examination; NC, normal control; TMT-A, Trail Making Test, part A; TMT-B, Trail Making Test, part B. 
The results from language and praxis domains did not reveal significant differences during the period of 1 year.

The patients were classified into three subgroups at baseline: two patients (3\%) with NCI, $57 \mathrm{MCI}$ patients (77\%), and 15 patients with dementia (20\%). At 1 year follow-up investigations, the results revealed $15 \mathrm{NCI}$ patients $(20 \%)$, $30 \mathrm{MCI}$ patients (41\%), and 29 patients with dementia (39\%). Eleven patients were lost for follow-up at 12 months.

We followed the patients' subgroups at baseline. The proportion of patients improving or developing dementia and the proportion of patients meeting NCI, MCI, and dementia were determined. From the dementia group, four patients improved their cognitive status - three patients to MCI level and one to $\mathrm{NCI}$ - and eight patients remained with dementia. From the MCI patients group, 27 patients remained in MCI, 18 patients became demented, and 12 patients improved to NCI.

\section{Subsequent IST subgroups}

The patients were classified into two subgroups according to their IST score at the baseline. IST score of 28 was used as cutoff corresponding to the subjects' educational level. ${ }^{20}$ Table 1 presents the distribution of demographic and clinical characteristics according to the IST score subgroups. The number of patients with IST scores (IST >28) within the NL was $46(44.1 \%)$ and those at risk of cognitive decline (IST $\leq 28$ ) was 39 (45.9\%). Furthermore, the patients at risk with IST $\leq 28$ coincided with the same group of patients with IST performance $<2.0 \mathrm{SD}$ below the mean from the age-matched control population. Table 3 showed the results of neuropsychological assessments at baseline in patients divided by IST criterion.

\section{Neuropsychological assessments and IST groups}

To determine the profile of the follow-up exams on IST and trace the sensitivity of the IST division, a two-way rANOVA on two groups of patients as a between-subject variable and four scores/follow-up exams during 1 year as a within-subject variable was performed. A significant main effect of group factor $(F[1,72]=50.84, P<0.001)$ and of follow-up exams $(F[3,216]=3.61, P<0.05)$ was obtained. Main effect of the group showed that patients at risk were having significantly lower IST scores ( mean =32) than NL patients (mean $=25$, Table 4). Most importantly, a significant interaction $(F[3,216]=5.85, P<0.001)$ was also obtained that suggested different pattern of improvement in IST score over time for both groups (Table 4). In particular, while there was no change in IST over time for NL patients, the patients at risk group showed significant
Table 3 Neuropsychological assessments in patients' subgroups divided by IST criterion

\begin{tabular}{|c|c|c|}
\hline Fifth day & $\begin{array}{l}\text { Stroke patients } \\
\text { (at risk) IST } \leq \mathbf{2 8}\end{array}$ & $\begin{array}{l}\text { Stroke patients } \\
(\mathrm{NL}) \text { IST }>\mathbf{2 8}\end{array}$ \\
\hline MMSE & $25.7(2.7)$ & $27.3(1.5)^{* *}$ \\
\hline - Orientation & $9.5(0.7)$ & $9.8(0.4)^{*}$ \\
\hline - Memory & $5.2(0.9)$ & $5.5(0.6)^{*}$ \\
\hline - Attention & $3.8(1.4)$ & $4.3(0.8) *$ \\
\hline - Language & $6.5(0.7)$ & $6.8(0.6)^{*}$ \\
\hline - Praxis & $0.7(0.4)$ & $0.8(0.4)$ \\
\hline IST & $23.5(4.6)$ & $32.5(2.7)^{* * * *}$ \\
\hline IADL (4 items) & $0.31(0.9)$ & $0.02(0.1)^{*}$ \\
\hline \multicolumn{3}{|c|}{ CERAD episodic memory } \\
\hline \multicolumn{3}{|c|}{ - Immediate recall } \\
\hline - I trial & $2.6(1.4)$ & $3.4(1.3)^{* *}$ \\
\hline - II trial & $4.4(1.4)$ & $5.7(1.6)^{* * *}$ \\
\hline - III trial & $5.2(1.6)$ & $6.6(1.3)^{* * *}$ \\
\hline - Delayed recall & $2.1(1.9)$ & $4.3(2.0)^{* * *}$ \\
\hline - Recognition & $15.3(3.0)$ & $17.7(2.2) * * *$ \\
\hline TMT-A & $116(43)$ & $84(44)^{* *}$ \\
\hline TMT-B & $248(62)$ & $176(66)^{* * *}$ \\
\hline BNT & $13.8(1.2)$ & $14.4(1.0)^{*}$ \\
\hline Figure copying & $10.0(1.2)$ & $10.4(1.0)$ \\
\hline GDS & $5.1(3.4)$ & $3.4(2.9)^{*}$ \\
\hline
\end{tabular}

Notes: Means are given with standard deviations in parentheses separately for patients divided by IST criterion. $t$-test compares scores between two groups of IST-divided patients. $* P<0.05$; $* * P<0.01$; $* * * P<0.00$ I.

Abbreviations: BNT, Boston Naming Test; CERAD, Consortium to Establish a Registry for Alzheimer's Disease; GDS, Geriatric Depression Scale; IADL, Instrumental Activities of Daily Living; IST, Isaacs Set Test; MMSE, mini-mental state examination; NL, normal limits; TMT-A, Trail Making Test, part A;TMT-B, Trail Making Test, part B.

improvement in IST score after the 6th month (from 23.5 to 26.7; $P<0.001$ ); however, significantly lower results of IST scores remained even at 12 months $(26.3$, Table 4). In the following analyses, two groups based on IST division were taken as a between-subject variable and as a within-subject variable: different neuropsychological measures taken over time. Despite highly significant main effect of patients' subtypes on MMSE scale $(F[1,72]=17.17, P<0.001)$, no main effects of follow-up exams or an interaction were obtained (all $P>0.1$ ). Thus, MMSE scores did not change over time. Further, IST measure showed significant difference between the patients' groups in memory $(F[1,72]=12.21, P<0.001)$ and delayed response $(F[1,72]=15.40, P<0.001)$; patients at risk always showed worse improvement than NL patients. The interactions were not significant (all $P_{\mathrm{s}}>0.1$ ). Word recognition, however, did show dynamic sensitivity to the IST measure. The analysis obtained significant main effects $(F[1,72]=20.35, P<0.001 ; F[3,216]=20.89, P<0.001)$ and an interaction $(F[3,216]=3.42 ; \mathrm{KP}<0.05)$. The interaction suggested (Table 4) no improvement in NL patients and a sharp improvement in the patients at risk $(P<0.001)$ starting from the second measure in time. Finally, rANOVA on 
Table 4 Means of neuropsychological measures $\pm 95 \%$ confidence intervals (in parentheses) per condition

\begin{tabular}{|c|c|c|c|c|}
\hline \multirow[t]{2}{*}{ IST groups } & \multicolumn{4}{|c|}{ Measures in time } \\
\hline & 5th day & I st month & 6th month & I 2th month \\
\hline & IST & & & \\
\hline At risk & $23.5(1.5)$ & $25.1(2.1)$ & $26.7(1.8)$ & $26.3(2.1)$ \\
\hline \multirow[t]{2}{*}{ NL } & $32.6(0.8)$ & $31.4(1.3)$ & $32.3(1.5)$ & $31.5(1.5)$ \\
\hline & MMSE & & & \\
\hline At risk & $25.3(I . I)$ & $25.7(0.9)$ & $26.2(1.0)$ & $25.1(1.2)$ \\
\hline \multirow[t]{2}{*}{ NL } & $27.1(0.6)$ & $27.3(0.4)$ & $27.4(0.5)$ & $27.3(0.7)$ \\
\hline & Recognition & & & \\
\hline At risk & I5.3 (I.0) & $16.9(0.8)$ & I7.3 (0.8) & I7.3 (0.9) \\
\hline \multirow[t]{2}{*}{ NL } & $17.7(0.7)$ & $18.7(0.5)$ & $18.6(0.6)$ & $18.9(0.4)$ \\
\hline & Delayed rec & & & \\
\hline At risk & $2.1(0.6)$ & $3.4(0.8)$ & $3.9(0.8)$ & $4.1(0.9)$ \\
\hline \multirow[t]{2}{*}{ NL } & $4.3(0.6)$ & $5.2(0.8)$ & $5.3(0.6)$ & $6.0(0.7)$ \\
\hline & IADL & & & \\
\hline At risk & $0.3(0.3)$ & $0.9(0.4)$ & $0.8(0.4)$ & $\mathrm{I} .4(0.8)$ \\
\hline NL & $0.0(0.0)$ & $0.4(0.2)$ & $0.3(0.2)$ & $0.3(0.2)$ \\
\hline
\end{tabular}

Notes: NL group: the patients with IST score within the NL (IST >28). At risk group: those at risk of cognitive decline with IST score $\leq 28$.

Abbreviations: IADL, Instrumental Activities of Daily Living; IST, Isaacs Set Test; MMSE, mini-mental state examination; NL, normal limits.

IADL showed both significant main effects $(F[1,72]=11.71$, $P<0.01 ; F[3,216]=10.09, P<0.001)$ and interaction $(F[3,216]=4.12, P<0.01)$. The interaction showed that after 1 year of stroke, patients at risk were less able to take care of themselves $(P<0.001)$ than the NL patients (Table 4$)$.

\section{Neuroimaging measures}

First, a difference between the two groups in neuroimaging measures was tested. One-way ANOVA was run on the difference between the IST groups and the neuroimaging measures. The results showed that patients at risk have significantly more hippocampal atrophy $(F[1,83]=8.32$, $P<0.01)$ and basal ganglia lesions $(F[1,82]=5.78, P<0.05)$ than NL patients (Table 5).

Second, simultaneous multiple regressions were carried out with three neuroimaging predictors (PVC, basal ganglia lesions, and hippocampal atrophy on each neuropsychological measure; Table 6). The analyses showed hippocampal atrophy as a very stable predictor of cognitive impairment in almost all measures. Basal ganglia lesions were important only in time and language measures. None of the other predictors contributed to the variance.

Table 5 Means of neuroimaging measures $\pm 95 \%$ confidence intervals (in parentheses) per IST group

\begin{tabular}{llll}
\hline IST groups & $\begin{array}{l}\text { Hippocampal } \\
\text { atrophy }\end{array}$ & $\begin{array}{l}\text { Basal ganglia } \\
\text { lesions }\end{array}$ & PVC \\
\hline At risk & $1.2(0.4)$ & $8.8(1.7)$ & $2.8(0.4)$ \\
NL & $0.6(0.2)$ & $6.4(1.2)$ & $2.3(0.4)$ \\
\hline
\end{tabular}

Notes: NL group: the patients with IST score within the NL (IST >28). At risk group: those at risk of cognitive decline with IST score $\leq 28$.

Abbreviations: IST, Isaacs Set Test; NL, normal limits; PVC, periventricular change.

\section{Predictive value of baseline cognitive} assessment for subsequent dementia

We compared baseline neuropsychological performances and neuroimaging markers in three patient subgroups (NCI, MCI, and dementia) at 12th month. The results revealed that there were significant differences in hippocampal atrophy $(P<0.05 / P<0.0001)$, IST $(P<0.01 / P<0.01)$, TMT-A $(P<0.01 / P<0.001)$, TMT-B $(P<0.001 / P<0.001)$, delayed recall $(P<0.001 / P<0.001)$, recognition $(P<0.001 / P<0.01)$, immediate recall $(P<0.001 / P<0.01)$, memory subscale of MMSE $(P<0.01 / P<0.01)$, and MMSE $(P<0.001 / P<0.01)$ between the dementia group and the other groups $(\mathrm{MCI} / \mathrm{NCI})$ at 1 year follow-up.

\section{Discussion}

The objective of our study was to evaluate the delayed changes in cognition in poststroke patients and their relationship to the neuropsychological and neuroimaging markers measured during the acute poststroke phase in first-ever stroke patients. The results of this study revealed that dementia affected up to $20 \%$ of patients at the acute phase of stroke and $39 \%$ of stroke survivors after 1 year. The frequency of dementia and cognitive impairment after cerebrovascular stroke varies between studies. Ballard et $\mathrm{al}^{3}$ found that $9 \%$ of patients developed incident dementia over the 1-year follow-up period. Madureira et $\mathrm{a}^{21}$ found the frequency of PSD 3 months after stroke to be only $6 \%$. However, Khedr et al ${ }^{4}$ revealed that dementia affects up to $21 \%$ of stroke survivors 3 months after stroke. In a subsequent hospitalized cohort studied 3 months after stroke, PSD was found in $26 \%(66 / 251) .{ }^{22}$ Jonkman et $\mathrm{al}^{23}$ reported that the incidence of cognitive impairment 3 months after stroke 
Table 6 Simultaneous multiple regression analyses on neuropsychological measures (12th month) with neuroimaging predictors

\begin{tabular}{|c|c|c|c|c|c|c|c|c|c|}
\hline \multirow{2}{*}{$\begin{array}{l}\text { Neuropsychological } \\
\text { measures at I 2th month }\end{array}$} & \multicolumn{3}{|c|}{ Hippocampal atrophy } & \multicolumn{3}{|c|}{ Basal ganglia lesions } & \multicolumn{3}{|c|}{ PVCs } \\
\hline & $\beta$ & $t$ & $P$-value & $\beta$ & $t$ & $P$-value & $\beta$ & $t$ & $P$-value \\
\hline MMSE & -0.3 & -2.2 & 0.03 & -0.2 & -1.9 & 0.07 & 0.0 & 0.1 & 0.94 \\
\hline MMSE (orientation) & -0.2 & -1.6 & 0.1 & -0.2 & -1.3 & 0.2 & -0.0 & -0.3 & 0.8 \\
\hline MMSE (attention) & -0.3 & -2.4 & 0.01 & -0.1 & -1.2 & 0.2 & -0.0 & -0.0 & 0.9 \\
\hline MMSE (memory) & -0.3 & -2.4 & 0.02 & -0.1 & -0.5 & 0.6 & 0.1 & 0.8 & 0.4 \\
\hline MMSE (language) & 0.1 & I.I & 0.3 & -0.3 & -2.4 & 0.02 & -0.1 & -0.8 & 0.4 \\
\hline MMSE (praxis) & -0.1 & -1.0 & 0.3 & -0.1 & -0.6 & 0.6 & 0.1 & 0.7 & 0.5 \\
\hline IST & -0.4 & -3.3 & 0.001 & -0.2 & -1.3 & 0.19 & -0.1 & -1.0 & 0.31 \\
\hline IADL & 0.3 & 2.5 & 0.01 & 0.0 & 0.2 & 0.83 & -0.1 & -0.4 & 0.7 \\
\hline Delayed recall & -0.4 & -3.8 & 0.00 & 0.0 & 0.3 & 0.7 & -0.1 & 0.4 & 0.7 \\
\hline Recognition & -0.3 & -2.9 & 0.005 & -0.0 & -0.3 & 0.7 & -0.2 & -1.2 & 0.2 \\
\hline TMT-B & 0.3 & 2.7 & 0.008 & 0.3 & 2.5 & 0.01 & 0.2 & 1.6 & 0.1 \\
\hline TMT-A & 0.3 & 2.8 & 0.007 & 0.3 & 2.7 & 0.01 & 0.1 & 1.1 & 0.3 \\
\hline
\end{tabular}

Note: For each predictor, the $\beta, t$ statistic, and $P$-value are reported.

Abbreviations: $\beta$, beta weight; IADL, Instrumental Activities of Daily Living; IST, Isaacs Set Test; MMSE, mini-mental state examination; PVCs, periventricular changes; TMT-A, Trail Making Test, part A; TMT-B, Trail Making Test, part B.

was $35 \%-37 \%$. In community-based studies, the prevalence of PSD is approximately $30 \%$ and the incidence of new-onset dementia after stroke increases from $7 \%$ after 1 year to $48 \%$ after 25 years. ${ }^{6}$ Our finding falls between these extreme values. Béjot et $\mathrm{al}^{24}$ examined 3,948 first-ever stroke patients over 24 years. The presence of dementia during the 1 st month after stroke was diagnosed in $20.4 \%$ of patients. They showed that the prevalence of PSD associated with lacunar stroke was seven times higher than that in intracerebral hemorrhage but declined over time as did prestroke antihypertensive medication. The difference of prevalence of PSD among various studies depends on the study design and the population studied, the criteria used for the diagnosis, neuroimaging markers, vascular risk factors, the time interval between the stroke and the neuropsychological assessment, and length of follow-up.,20,22-27

Although in our study $36.4 \%$ of patients had mild depression, this was not significantly associated with poststroke cognitive impairment. In general, depression is a common consequence of stroke with a reported frequency of up to $65 \%$ of all patients. ${ }^{4}$

While the prototypic cognitive changes preceding dementia of the Alzheimer type consist of impaired episodic memory, vascular dementia is frequently associated with executive dysfunction. However, many patients are impaired in several cognitive domains, probably reflecting the site and extent of anatomical lesions. ${ }^{8}$ In our study, attention, executive functions, processing speed, memory, and orientation were significantly involved in poststroke cognitive impairment. In agreement with our data, a prospective populationbased study ${ }^{28}$ showed that executive dysfunction as evidenced by verbal fluency and TMT-B performance was significantly associated with early IADL deficits and subsequent dementia. In addition, Oksala et $\mathrm{al}^{29}$ showed that in patients with ischemic stroke, cognitive impairment, particularly in executive functions and visuospatial/constructional abilities, is related to poor survival. It should be noted, however, that it is still unclear whether and to what extent deficits in specific cognitive domains predict delayed cognitive and functional decline in patients with ischemic stroke. Identifying poststroke cognitive impairment at an early stage might increase the possibility of reducing or even preventing further cognitive decline. Moreover, an early diagnosis of specific cognitive deficits, such as memory impairment or executive dysfunction, could be of great importance in determining the effect on daily functioning. ${ }^{7,30}$

The results of the present study revealed that neurological and daily living activity outcome 1 year after stroke in cognitively intact patients is significantly higher than in patients at risk measured by IST at baseline. We found that IST as a screening test could be a reliable and quick tool for poststroke cognitive impairment evaluation and delayed cognitive and neurological outcome. Our results, however, showed that MMSE did not appear to be a significant predictor. Although the MMSE is one of the most frequently used cognitive tests in research and clinical practice, Al-Qazzaz et a ${ }^{31}$ and Nys et $\mathrm{al}^{32}$ found that the MMSE is an invalid screening tool for discriminating between cognitively intact and impaired stroke patients. In agreement with this finding, a study focusing on young (18-47 years old) stroke patients also suggested that the MMSE is not able to detect a neuropsychological deficit. ${ }^{33}$ Thus, studies confirm that MMSE is not always an effective tool of measuring neuropsychological deficits.

It should be noted that there is evidence suggesting that assessing additional cognitive functions, such as executive 
functioning, abstract reasoning, and speed of information processing, might improve the screening, because impairments in these areas are often significant predictors of both cognitive and functional impairment over the long term. However, extensive neuropsychological testing is time consuming and can be exhausting for the patient. Thus, short appropriate cognitive screening seems to be the obvious best way to detect cognitive impairments in the early days poststroke. ${ }^{7}$ The Tel Aviv Brain Acute Stroke Cohort (TABASCO) study is an ongoing, prospective cohort study that plan to recruit approximately 1,125 consecutive first-ever mild to moderate stroke patients. Using a computerized battery of neuropsychological tests for memory, attention, and executive functions (MindStreams ${ }^{\circledR}$ ) in 545 poststroke patients demonstrated that either of the modalities alone is sensitive enough for identifying subtle cognitive impairment. ${ }^{34}$

The role of neuroimaging markers including PVCs and MTLA as a contributor to poststroke cognitive decline is still controversial. ${ }^{8}$ In our study, hippocampal atrophy assessed by visual scale is the strongest predictor of cognitive impairment and dementia outcome, even in poststroke patients. Poststroke patients at risk of cognitive impairment showed significantly more basal ganglia lesions in comparison with cognitively intact stroke patients. Although CT is less accurate than MRI in demonstrating the degree of PVCs, a previous study showed a good agreement between grading of changes on these scales on CT and MRI. ${ }^{13}$

PSD is well documented in patients with extensive subcortical white matter lesions. ${ }^{24}$ However, $30 \%$ of normal individuals over 65 years of age have moderate white matter hyperintensities (WMH) on MRI and 7\% have severe WMH. ${ }^{8,35}$ Stebbins et al demonstrated significant gray matter volume reductions, mostly in thalamus in patients with stroke with one or more cognitive domain impairments compared with patients with stroke without cognitive impairment using voxel-based optimized morphometry techniques. ${ }^{36} \mathrm{~A}$ recent study showed that in the whole stroke group, putamen lacunes were correlated with impairment in memory, while WMH and hippocampal volume both correlated with global dysfunction. ${ }^{37}$ Another study found that delayed PSD in elderly stroke survivors is associated with MTLA. ${ }^{38}$ Hénon et $\mathrm{al}^{27}$ showed that patients who had a stroke and MTLA more frequently develop dementia than patients without MTLA, but their study does not suggest that MTLA independently contributes to dementia. Firbank et $\mathrm{al}^{39}$ found that medial temporal atrophy rather than WMH predict cognitive decline in stroke survivors, suggesting a greater role for Alzheimer's type than vascular pathology in delayed cognitive impairment after stroke. In contrast, recent studies revealed that midlife vascular risk factors are associated with an increased rate of global and hippocampal atrophy. Moreover, hippocampal atrophy is presented not only in Alzheimer's disease but also in cerebrovascular disease and subcortical vascular dementia. These data could explain cognitive deficits related to hippocampal atrophy in a pure vascular process. ${ }^{40,41}$

Strengths of our study included the prospective and consecutive recruitment of a well-characterized cohort of Bulgarian stroke patients and controls. The patients were thoroughly examined four times with neuropsychological sensitive battery for a period of 1 year.

The main limitations of the current study might be our small sample size for detecting longitudinal decline and use of CT instead of MRI for assessing the neuroradiological markers. However, there is evidence of excellent intraobserver agreement between CT and MRI for medial temporal atrophy and WMC with published visual rating scales. ${ }^{13}$

\section{Conclusion}

Executive functioning deficit appears to have a predictive power for cognitive impairment progression. IST as a screening test has the potential to be a reliable and quick tool for poststroke cognitive impairment evaluation and delayed cognitive and neurological outcome. Hippocampal atrophy is the strongest predictor for cognitive impairment outcome, even in poststroke cognitive impairment. Identifying the neuropsychological and neuroimaging markers for delayed cognitive and functional decline may set the stage for better poststroke management and lead to important therapeutic implications.

\section{Acknowledgments}

The authors are grateful to the patients and their caregivers for cooperating in this research study.

\section{Disclosure}

The authors report no conflicts of interest in this work.

\section{References}

1. Desmond DW, Moroney JT, Sano M, Stern Y. Recovery of cognitive function after stroke. Stroke. 1996;27(10):1798-1803.

2. Pohjasvaara T, Erkinjuntti T, Yilkoski R, Hietanen M, Vataja R, Kaste M. Clinical determinants of poststroke dementia. Stroke. 1998;29(1):75-81.

3. Ballard C, Rowan E, Stephens S, Kalaria R, Kenny RA. Prospective follow-up study between 3 and 15 months after stroke: improvements and decline in cognitive function among dementia-free stroke survivors $>75$ years of age. Stroke. 2003;34(10):2440-2444.

4. Khedr EM, Hamed SA, El-Shereef HK, et al. Cognitive impairment after cerebrovascular stroke: Relationship to vascular risk factors. Neuropsychiatr Dis Treat. 2009;5:103-116.

5. Tham W, Auchus AP, Thong M, et al. Progression of cognitive impairment after stroke: one year results from a longitudinal study of Singaporean stroke patients. J Neurol Sci. 2002;203-204:49-52. 
6. Leys D, Hénon H, Mackowiak-Cordoliani MA, Pasquier F. Poststroke dementia. Lancet Neurol. 2005;4(11):752-759.

7. van der Zwaluw CS, Valentijn SA, Nieuwenhuis-Mark R, Rasquin SM, van Heugten CM. Cognitive functioning in the acute phase poststroke: a predictor of discharge destination? J Stroke Cerebrovasc Dis. 2011; 20(6):549-555.

8. Ben Assayag E, Korczyn AD, Giladi N, et al. Predictors for poststroke outcomes: the Tel Aviv Brain Acute Stroke Cohort (TABASCO) study protocol. Int J Stroke. 2012;7(4):341-347.

9. Brott T, Adams HP Jr, Olinger CP, et al. Measurements of acute cerebral infarction: a clinical examination scale. Stroke. 1989;20(7):864-870.

10. Scheltens P, Leys D, Barkhof F, et al. Atrophy of medial temporal lobes on MRI in "probable" Alzheimer's disease and normal aging: diagnostic value and neuropsychological correlates. J Neurol Neurosurg Psychiatry. 1992;55(10):967-972.

11. Scheltens P, Launer LJ, Barkhof F, Weinstein HC, van Gool WA. Visual assessment of medial temporal lobe atrophy on magnetic resonance imaging: interobserver reliability. J Neurol. 1995;242(9):557-560.

12. Scheltens P, Barkhof F, Leys D, et al. A semiquantative rating scale for the assessment of signal hyperintensities on magnetic resonance imaging. J Neurol Sci. 1993;114(1):7-12.

13. Wattjes MP, Henneman WJ, van der Flier WM, et al. Diagnostic imaging of patients in a memory clinic: comparison of MR imaging and 64-detector row CT. Radiology. 2009;253(1):174-183.

14. Strauss E, Sherman EMS, Spreen O. A Compendium of Neuropsychological Tests. 3rd ed. New York: Oxford University Press; 2006.

15. Isaac B, Kennie AT. The Set test as an aid to the detection of dementia in old people. Br J Psychiatry. 1973;123(575):467-470.

16. Amieva H, Jacqmin-Gadda H, Orgogozo JM, et al. The 9 year cognitive decline before dementia of the Alzheimer type: a prospective population-based study. Brain. 2005;128(Pt 5):1093-1101.

17. Barberger-Gateau P, Dartigues JF, Letennuer L. Four Instrumental Activities of Daily Living score as a predictor of one-year incident dementia. Age Ageing. 1993;22(6):457-463.

18. Winblad B, Palmer K, Kivipelto M, et al. Mild cognitive impairment beyond controversies, towards a consensus: report of the International Working Group on Mild Cognitive Impairment. J Intern Med. 2004; 256(3):240-246.

19. American Psychiatric Association. Diagnostic and Statistical Manual of Mental Disorders. 4th ed. Washington, DC: American Psychiatric Association; 1994.

20. Raycheva M, Stoyanova K, Janian A, et al. [Validation of Bulgarian versions of Mini-Mental State Examination и Isaac's Set Test as screening assessments for cognitive impairments in elderly.] Psychological Assessments. 2013;1-2:139-156. Bulgarian.

21. Madureira S, Guerreiro M, Ferro JM. Dementia and cognitive impairment three months after stroke. Eur J Neurol. 2001;8(6):621-627.

22. Tatemichi TK, Desmond DW, Mayeux R, et al. Dementia after stroke: baseline frequency, risks, and clinical features in a hospitalized cohort. Neurology. 1992;42(6):1185-1193.

23. Jonkman EJ, de Weerd AW, Vrijens NL. Quality of life after first ischemic stroke. Long term development and correlation with changes in neurological deficit, mood and cognitive impairment. Acta Neurol Scand. 1998;98(3):169-175.

24. Béjot Y, Aboa-Eboulé C, Durier J, et al. Prevalence of early dementia after first-ever stroke: a 24-year population-based study. Stroke. 2011;42(3): 607-612.

Neuropsychiatric Disease and Treatment

\section{Publish your work in this journal}

Neuropsychiatric Disease and Treatment is an international, peerreviewed journal of clinical therapeutics and pharmacology focusing on concise rapid reporting of clinical or pre-clinical studies on a range of neuropsychiatric and neurological disorders. This journal is indexed on PubMed Central, the 'PsycINFO' database and CAS,
25. Pohjasvaara T, Mäntylä R, Ylikoski R, Kaste M, Erkinjuntti T. Comparison of different clinical criteria (DSM-III, ADDTC, ICD-10, NINDSAIREN, DSM-IV) for the diagnosis of vascular dementia. National Institute of Neurological Disorders and Stroke-Association Internationale pour la Rechherche et I'Enseignement en Neurosciences. Stroke. 2000;31(12):2952-2957.

26. Tatemichi TK, Foulkes MA, Mohr JP, et al. Dementia in stroke survivors in the Stroke Data Bank cohort: prevalence, incidence, risk factors, and computed tomographic findings. Stroke. 1990;21(6):858-866.

27. Hénon H, Pasquier F, Leys D. Poststroke dementia. Cerebrovasc Dis. 2006; 22(1):61-70.

28. Pérès K, Helmer C, Amieva $\mathrm{H}$, et al. Natural history of decline in instrumental activities of daily living performance over the 10 years preceding the clinical diagnosis of dementia: a prospective population-based study. J Am Geriatr Soc. 2008;56:37-44.

29. Oksala NK, Jokinen H, Melkas S, et al. Cognitive impairment predicts poststroke death in long-term follow-up. J Neurol Neurosurg Psychiatry. 2009;80(11):1230-1235.

30. Nys GM, van Zandvoort MJ, de Kort PL, Jansen BP, de Haan EH, Kappelle LJ. Cognitive disorders in acute stroke: prevalence and clinical determinants. Cerebrovasc Dis. 2007;23(5-6):408-416.

31. Al-Qazzaz NK, Ali SH, Ahmad SA, Islam S. Cognitive assessments for the early diagnosis of dementia after stroke. Neuropsychiatr Dis Treat. 2014; 10:1743-1751.

32. Nys GM, van Zandvoort MJ, de Kort PL, Jansen BP, Kappelle LJ, de Haan EH. Restrictions of the Mini-Mental State Examination in acute stroke. Arch Clin Neuropsychol. 2005;20(5):623-629.

33. Cao M, Ferrari M, Patella R, Marra C, Rasura M. Neuropsychological findings in young-adult stroke patients. Arch Clin Neuropsychol. 2007;22(2): 133-142.

34. Shopin L, Shenhar-Tsarfaty S, Ben Assayag E, et al. Cognitive assessment in proximity to acute ischemic stroke/transient ischemic attack: comparison of the montreal cognitive assessment test and mindstreams computerized cognitive assessment battery. Dement Geriatr Cogn Disord. 2013;36(1-2):36-42.

35. Hunt AL, Orrison WW, Yeo RA, et al. Clinical significance of MRI white matter lesions in the elderly. Neurology. 1989;39(11):1470-1474.

36. Stebbins GT, Nyenhuis DL, Wang C, et al. Gray matter atrophy in patients with ischemic stroke with cognitive impairment. Stroke. 2008; 39(3):785-793.

37. Lopes MA, Firbank MJ, Widdrington M, Blamire AM, Kalaria RN, O'Brien JT. Post-stroke dementia: the contribution of thalamus and basal ganglia changes. Int Psychogeriatr. 2012;24(4):568-576.

38. Gemmell E, Bosomworth H, Allan L, et al. Hippocampal neuronal atrophy and cognitive function in delayed poststroke and aging-related dementias. Stroke. 2012;43(3):808-814.

39. Firbank MJ, Burton EJ, Barber R, et al. Medial temporal atrophy rather than white matter hyperintensities predict cognitive decline in stroke survivors. Neurobiol Aging. 2007;28(11):1664-1669.

40. Debette S, Seshadri S, Beiser A, et al. Midlife vascular risk factor exposure accelerates structural brain aging and cognitive decline. Neurology. 2011; 77(5):461-468.

41. van de Pol L, Gertz HJ, Scheltens P, Wolf H. Hippocampal atrophy in subcortical vascular dementia. Neurodegener Dis. 2011;8(6):465-469.

and is the official journal of The International Neuropsychiatric Association (INA). The manuscript management system is completely online and includes a very quick and fair peer-review system, which is all easy to use. Visit http://www.dovepress.com/testimonials.php to read real quotes from published authors. 